\title{
Le polissage sous contraintes
}

\author{
Emmanuel HUGOT \\ Aix Marseille Université - CNRS - Laboratoire d'Astrophysique de Marseille \\ emmanuel.hugot@lam.fr
}

La fabrication optique est depuis toujours un thème riche en recherche et développement, afin de répondre aux objectifs scientifiques des grandes infrastructures de recherche en physique et astrophysique. Surfaces plus précises, actives, rugosité sub-nanométrique, moins de défauts d'aspect, peu de hautes fréquences spatiales, minimisation des défauts sous les surfaces pour optimiser la tenue au flux laser, sont autant de défis à relever lors du polissage des surfaces optiques. Le polissage sous contraintes se place comme une méthode particulièrement efficace pour répondre à ces attentes, en permettant de produire des surfaces complexes tout en conservant un travail par outils pleine taille. Présentation.

'est dans son atelier de l'observatoire de Bergedorf près de Hambourg, où il est surnommé « l'Opticien », que le jeune Bernhardt Schmidt (1879-1935) conçoit la fameuse caméra grand champ qui portera son nom. Constituée d'un miroir primaire sphérique de $44 \mathrm{~cm}$ ouvert à $F / 1,75$, la première caméra de Schmidt offre un champ de vue de 7,5 degrés. Cette combinaison optique nécessite la compensation de l'aberration sphérique introduite par le miroir primaire, ce qui est réalisé par une lame correctrice d'entrée dont la forme spécifique, dite asphérique, est placée au niveau du rayon de courbure du primaire.

Schmidt était connu pour la grande qualité des pièces optiques taillées dans son atelier et notamment pour la superbe qualité de surface de ses lames correctrices asphériques. Son secret de fabrication ne fut découvert qu'après sa mort lorsque, pénétrant dans son atelier, ses collaborateurs découvrirent différents systèmes de chambres de pression reliés à ses machines de polissage. L'artisanartiste fut le pionnier de l'optique active, le premier à utiliser les déformations par dépression des pièces optiques lors de la fabrication pour réaliser les surfaces complexes désirées à l'aide des techniques traditionnelles. Le polissage sous contraintes était inventé.

Le principe est simple, l'idée en est d'autant plus géniale : à l'aide de systèmes de pression, les lames sont déformées selon l'inverse de leur forme finale. La réalisation d'un polissage plan ou sphérique va retirer l'excédent de matière correspondant à la déformation introduite. En fin de polissage, lors de la relâche de la pression, la pièce optique devient asphérique et bénéficie de l'excellente qualité de surface d'un polissage classique par outil pleine taille, méthode utilisée par les hommes de l'art. Cette méthode extrêmement performante évite d'utiliser le polissage par retouches locales, long et coûteux, et dont le résultat est dégradé par les traces d'outils laissées sur la surface optique.

Appelée «polissage sous contraintes », cette méthode performante nécessite néanmoins une maîtrise parfaite des différents paramètres et des différentes étapes de fabrication. L'élément primordial est la connaissance des déformations des pièces optiques qui fait appel à la théorie de l'élasticité. Les développements théoriques permettent de déduire les points de fonctionnement, c'est-à-dire les géométries des substrats de miroirs et les cas de charge associés, en fonction de la forme finale souhaitée sur la surface optique.

La démonstration la plus saisissante a été réalisée à partir des années 1980 par L'université d'Arizona et le célèbre Steward Observatory Mirror Lab. À cette époque, le groupe de Jerry Nelson développe une

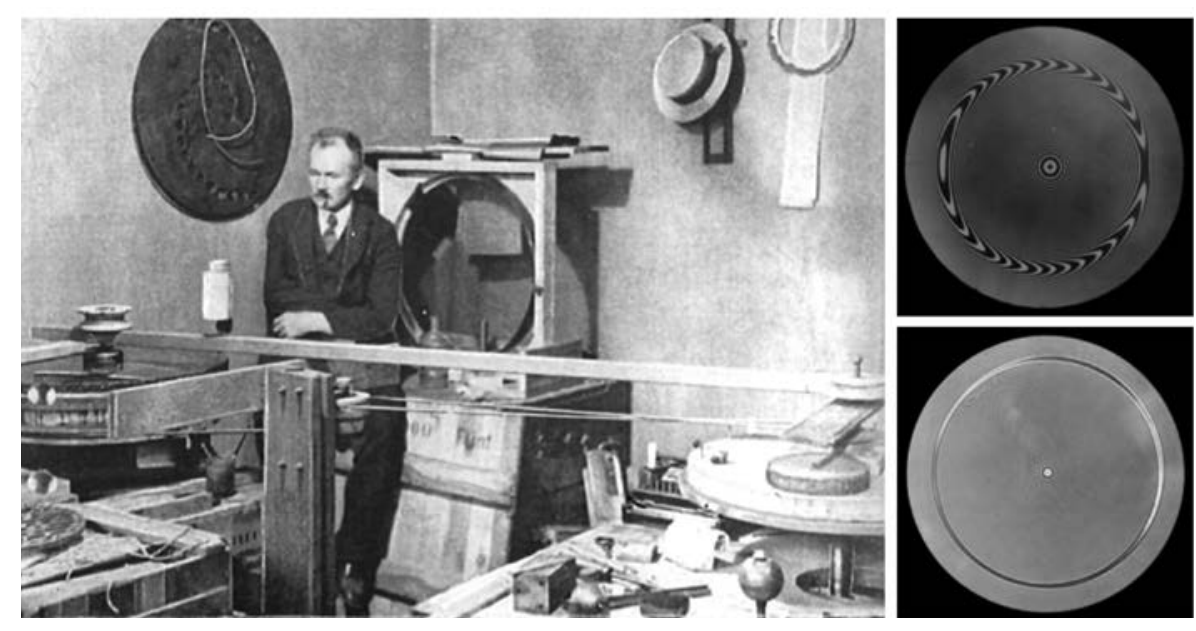

Figure 1. Bernhardt Schmidt dans son atelier à Bergedorf. Interférogrammes de lames de Schmidt obtenues par taille sous dépression. La qualité de polissage de ces surfaces est démontrée par la régularité des franges d'interférence obtenues au Laboratoire d'Optique de l'Observatoire de Marseille par Gérard Lemaitre. 
méthode de fabrication de paraboles hors axe par le biais du polissage sous contraintes, et propose de lancer la fabrication d'un télescope de grand diamètre en utilisant la segmentation des miroirs primaires. Les jumeaux Keck verront leur première lumière quelques années plus tard sur le Mauna Kea à Hawaii. Ces deux télescopes, qui comptent toujours parmi les trois plus grands au monde, possèdent un miroir primaire composé de 36 segments hexagonaux d'1,8 mètre, placés bout à bout et alignés pour reproduire une surface parabolique. Chacun des segments est une portion de parabole hors axe, surface asphérique dont la déformation est fonction de sa distance à l'axe optique.

En fonctionnement depuis plus de 20 ans, I'Observatoire du Keck avec ses deux télescopes aura démontré une série de ruptures technologiques qui vont permettre l'émergence de projets encore plus impressionnants : les futurs Extremely Large Telescopes (ELTs). Ces télescopes possèderont des diamètres de $24 \mathrm{~m}, 30 \mathrm{~m}$ et $39 \mathrm{~m}$, soit 2 à 4 fois supérieurs aux plus grands télescopes actuellement en service. Mais avant d'aborder ces projets ambitieux, faisons un détour par l'instrumentation astrophysique opérationnelle sur les grands télescopes actuels.

\section{L'imagerie directe d'exoplanètes : un défi optique}

L'un des sujets d'astronomie les plus passionnants du $21^{\mathrm{e}}$ siècle est sans doute la recherche de planètes extrasolaires, planètes orbitant autour d'étoiles autres que le Soleil. Découvrir d'autres mondes, en obtenir des images et caractériser la composition de leur atmosphère constituent un premier pas vers la réponse à la question fondamentale : sommes-nous seuls dans I'Univers?

Très concrètement, la détection, l'imagerie et la caractérisation d'exoplanètes sont autant de défis technologiques qui mobilisent une grande partie de la communauté des astronomes et ingénieurs. En effet, non seulement les planètes sont très proches de leurs étoiles hôtes, mais leur luminosité est environ 1 million à 1 milliard de fois plus faible que celle de leur étoile. Obtenir l'image d'une planète demande la combinaison de deux technologies de pointe : l'imagerie à haute résolution angulaire et l'imagerie à haut contraste.

L'imagerie à haute résolution angulaire depuis la Terre est réalisée à l'aide de systèmes complexes d'optique adaptative. Cette méthode utilise des miroirs déformables, qui corrigent en temps réel l'effet de flou créé par la propagation de la lumière à travers l'atmosphère terrestre. Les miroirs déformables sont composés d'une membrane réfléchissante, dont la forme est contrôlée par une batterie d'actionneurs qui vont compenser les variations de la turbulence atmosphérique. Cependant, ces miroirs ne peuvent pas corriger de défauts plus petits que l'espace entre deux actionneurs. Afin d'obtenir la meilleure qualité de correction, les différentes surfaces optiques de relai ne doivent pas comporter de défauts plus petits que cet espacement. C'est à ce niveau que le polissage sous contraintes intervient, grâce à l'excellente qualité de surface obtenue. Ne pas générer de défauts locaux, cela évite d'avoir à les corriger ! Le LAM a donc réalisé, au cours des dernières années, des surfaces complexes suivant cette méthode, afin d'être utilisées sur I'Optique Adaptative Extrême de l'instrument imageur d'exoplanètes VLT-SPHERE qui produit aujourd'hui des observations de qualité inégalée.

L'imagerie à haut contraste utilise notamment la coronographie. L'une des principales limitations de cette technique est la présence de speckles quasi-statiques résiduels provenant de la phase résiduelle atmosphérique et des aberrations instrumentales non-corrigées par optique adaptative. Au-delà des différentes méthodes de réduction du bruit de speckles proposées, par exemple par imagerie différentielle, la première marge de progression se situe au cœur-même des instruments, avec la minimisation des erreurs instrumentales.

\section{L'approche industrielle : la production de masse}

Les performances des optiques polies sous contraintes répondent aux exigences de l'imagerie directe d'exoplanètes. Cependant, la puissance de cette méthode permet également d'aborder des problématiques industrielles, notamment sur les aspects de production de masse. Cette problématique est étudiée de près pour proposer une méthode abordable de réalisation des miroirs primaires géants des futurs observatoires.

Le télescope géant (E-ELT) de l'European Southern Observatory (ESO) aura un miroir primaire de 39 mètres de diamètre, composé de près de 800 segments hexagonaux de 1,425 $\mathrm{m}$ coin à coin. Le miroir étant quasi parabolique, la surface de chacun des segments varie en fonction de sa distance à l'axe optique. En considérant les symétries (six secteurs sur le miroir) et les segments jumeaux, les surfaces optiques se répartissent en 133 familles distinctes. Chacune de ces familles a une forme spécifique, le segment le plus déformé présentant une asphéricité de $150 \mu \mathrm{m}$.

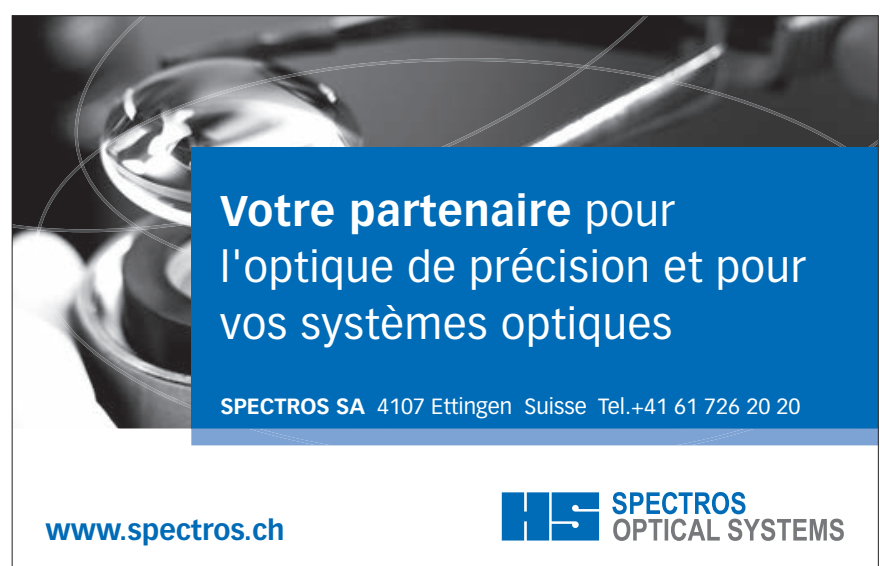



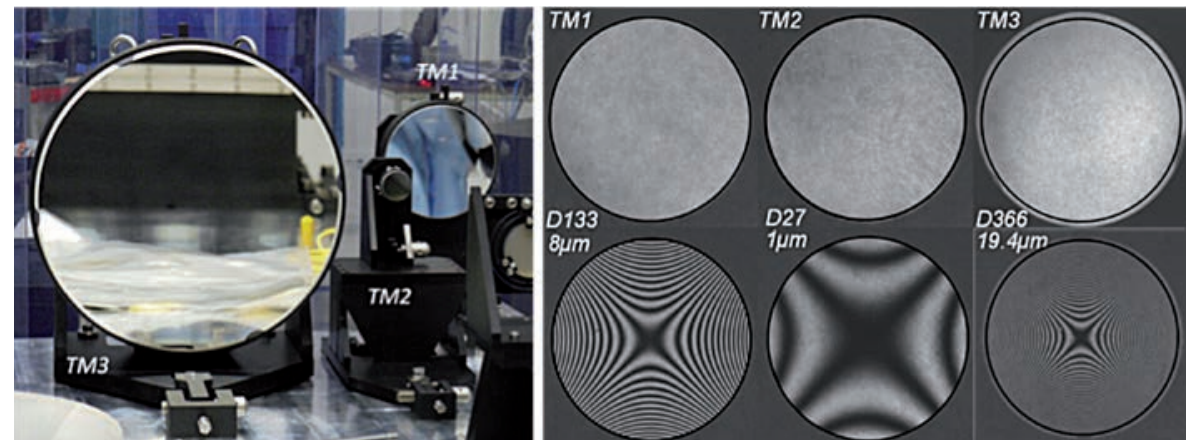

Figure 2. Les miroirs de relai hors d'axe de l'instrument VLT SPHERE, imageur de planètes extrasolaires, bénéficient de l'excellente qualité de surface obtenue par polissage sous contraintes. Les interférogrammes du haut correspondent à la qualité en sortie de polissage, les interférogrammes du bas sont les figures astigmates après la relaxation des contraintes. Le plus grand miroir (diamètre $366 \mathrm{~mm}$ ) possède une asphéricité (déviation par rapport à la sphère de polissage) de 19,4 um qui a été réalisée avec une précision de $\delta=22 \mathrm{~nm}$ RMS. Les autres miroirs ont une qualité de moins de $\delta=10 \mathrm{~nm}$ RMS. Une phase de superpoli a été appliquée, permettant de réduire la rugosité des surfaces à moins de $\sigma=0,5 \mathrm{~nm} R M S$.

C'est à partir d'ici que les choses se corsent : la spécification sur l'ensemble des segments est constante à $\delta=12 \mathrm{~nm}$ RMS sur l'ensemble de la surface optique. Atteindre une telle qualité sur de tels diamètres, sur un très grand nombre de pièces et dans un délai raisonnable devient un véritable casse-tête industriel. En regard du planning de réalisation de ces segments, le taux de production devra être d'un miroir par jour sur une période de 5 ans, en comptant la mise en place des moyens de production et la phase d'apprentissage. C'est le défi que souhaitent relever le LAM et Thales SESO, en développant une méthode de polissage sous contraintes qui permettra de réaliser avec un seul et unique outillage l'ensemble des familles et de réaliser leur polissage en moins de 30 heures par segment. Aujourd'hui sélectionnée pour la réalisation du Thirty Meter Telescope (TMT) américain, cette méthode est également pressentie au niveau Européen et sera soumise à l'ESO fin 2015 afin d'évaluer sa compétitivité.

\section{Champs d'applications}

Nous avons décrit quelques exemples d'applications du polissage sous contraintes ainsi que les performances et potentialités, mais il convient de préciser que cette méthode fait partie d'un domaine d'applications plus vaste que I'on nomme l'optique active. En effet, le

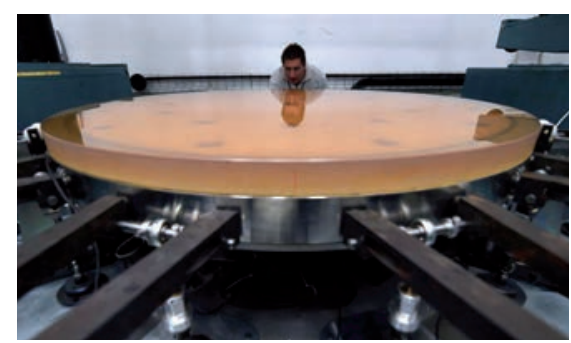

Figure 3. Le Laboratoire d'Astrophysique de Marseille est aujourd'hui chargé par l'ESO de développer et d'améliorer la méthode de polissage sous contraintes afin de rendre possible son utilisation pour la fabrication des quelque 800 segments qui composent le miroir primaire du futur télescope géant européen. L'image ci- dessus présente l'un de ces segments, d'un diamètre de 1,5 m, installé sur l'une des machines de polissage du LAM. Un système d'interférométrie permet de mesurer optiquement la déformation élastique donnée au miroir en Zerodur.

polissage sous contraintes est basé sur le contrôle des déformations de substrats à des précisions drastiques, et ceci avec une grande stabilité durant la phase de polissage.

Le contrôle des déformations des pièces optiques permet de générer des surfaces asphériques, mais permet aussi de corriger des aberrations optiques, en direct pendant les phases d'observation des télescopes. C'est une application bien connue des astronomes. L'optique active a acquis ses lettres de noblesse sur les plus grands observatoires du monde, suite à la mise en place par l'ESO du New
Technology Telescope (NTT) à I'Observatoire de La Silla-Paranal. La forme de son miroir primaire mince est contrôlée en permanence par un réseau d'actionneurs, permettant de conserver la qualité d'image optimale. Cette démonstration a permis de voir l'émergence des très grands miroirs primaires tels qu'utilisés au Very Large Telescope (VLT) et aux Observatoires Gemini Nord et Sud, respectivement dans I'hémisphère Nord (Hawaï) et I'hémisphère Sud (Chili). Le VLT I'illustre en particulier avec ses quatre miroirs primaires de 8,2 mètres de diamètre pour seulement $17,5 \mathrm{~cm}$ d'épaisseur, dont la forme doit être contrôlée et corrigée par optique active chaque minute, grâce à une batterie de 150 actionneurs en contact avec l'arrière du miroir.

Complémentaire de l'optique adaptative de par sa mise en œuvre, l'optique active se distingue clairement par les différents champs d'application qu'elle propose : le contrôle et la correction des défauts optiques intrinsèques à l'instrument, la compensation des chemins optiques dans l'instrument et la fabrication optique de très haute qualité. L'ajout de cette flexibilité dans les instruments permet de réduire leur complexité, mais aussi d'offrir de nouvelles fonctionnalités optiques, ce qui constitue un domaine de recherche à part entière, en changeant les paradigmes sur lesquels se basent les concepts optiques actuels. Affaire à suivre.

\section{Pour en savoir plus}

La page web du groupe de recherche: www.lam.fr/r-d-optics-instrumentation

G.R. Lemaitre, Astronomical Optics and Elasticity, Active Optics Methods (Springer, 2009)

G.R. Lemaitre, Appl. Opt. 11, 1630 (1972)

J. Nelson et al., Appl. Opt. 19, 2341 (1980)

R.N. Wilson, F. Franza, L. Noethe, J. Mod. Opt. 34, 485 (1987)

M. Ferrari, Astron. Astrophys. Suppl. Ser. 128 221 (1998)

R. Gilmozzi, J. Spyromilio, Messenger 127 11 (2007)

E. Hugot et al., Astronomy \& Astrophysics 538, A139 (2012)

E. Hugot et al., Applied Optics 48, 2932 (2009) J.L. Beuzit et al., SPHERE: a 'Planet Finder' instrument for the VLT, Proc. SPIE (2008) 